\title{
REPLY
}

\section{Reply to Comment on 'Giant suppression of shot-noise in double barrier resonant diode: a signature of coherent transport'}

\author{
V Ya Aleshkin ${ }^{1}$, L Reggiani $^{2}$, N V Alkeev $^{3}$, V E Lyubchenko ${ }^{3}$, \\ C N Ironside ${ }^{4}$, J M L Figueiredo ${ }^{4}$ and C R Stanley ${ }^{4}$ \\ ${ }^{1}$ Institute for Physics of Microstructures, Nizhny Novgorod GSP-105 603600, Russia \\ ${ }^{2}$ National Nanostructure Laboratory of INFM, Dipartimento di Ingegneria dell' Innovazione, \\ Università di Lecce, Via Arnesano s/n, 73100 Lecce, Italy \\ ${ }^{3}$ Institute of Radioengineering and Electronics, Russian Academy of Sciences, Moscow, \\ Russia \\ ${ }^{4}$ Department of Electronic and Electrical Engineering, University of Glasgow, Glasgow, UK
}

Received 27 October 2003

Published 23 March 2004

Online at stacks.iop.org/SST/19/665 (DOI: 10.1088/0268-1242/19/5/N04)

\begin{abstract}
Shot noise suppression below $1 / 2$ of the full Poisson value in double barrier resonant diodes is confirmed to be a signature of coherent rather than sequential tunnelling transport. We reply to the arguments of the previous comment which dispute the above claim. We anticipate the development of a rigorous theory that improves previous approaches without contradicting the essential findings we recently reported (Aleshkin et al 2003 Semicond. Sci. Technol. 18 L35).
\end{abstract}

\begin{abstract}
In a recent letter [1] we showed that suppression of shot-noise in resonant diodes below $1 / 2$ of full shot-noise value $2 q I$, with $q$ the unit charge and $I$ the steady current, is a signature of coherent against sequential tunnelling transport regime. The proof stems on the fact that the theoretical approach we developed for the coherent tunnelling regime predicts under suitable bias and temperature conditions a Fano factor below $1 / 2$ while the standard sequential tunnelling regime $[2,3]$ never allows for a value of the Fano factor below 1/2. These findings were in agreement with experiments.

In their comment, Blanter and Büttiker (BB) contest our theoretical discussion on the basis of the results they obtained on the same subject $[4,5]$ and which never allow for a Fano factor drop below $1 / 2$. To this purpose the comment reports several arguments on which we reply in order of appearance.

In our opinion, the sequential tunnelling regime cannot be interpreted as the semiclassical limit of the coherent tunnelling regime when considering current noise. The microscopic noise source in each regime are of a different nature, even if both correctly recover the Nyquist relation under equilibrium, because the sequential and coherent tunnelling are different mechanism for electron transfer in the resonant tunnelling
\end{abstract}

diode. In the coherent transport there is no scattering during tunnelling while scattering in the quantum well is essential for sequential tunnelling. Moreover, the increase in the number of channels does not introduce scattering. Consider the simple case when the Coulomb interaction is neglected. Then, in the sequential tunnelling regime the fluctuations are described by the differential rates controlling the relaxation of carrier number fluctuations inside the two terminal device through the contacts [3]. By contrast, in the coherent tunnelling regime the fluctuations are described by the transparency of the whole device through the partition noise mechanism. These two descriptions are inherently different and provide in general different results. Therefore, we do not agree on the expected equivalence of phase coherent and sequential results and on the estimated quantum corrections reported in the comment.

Concerning the details of calculations which lead to our equation (8) we note the following. We consider high voltage regime, $v>E_{F}$. However, to our knowledge neither explicitly nor implicitly we assumed $E_{0} \ll E_{F}$, and thus we believe that equation (8) is rigorously obtained. We add that a similar expression for the Fano factor was obtained in [6, 7] and 
that also in that case a Fano factor below 1/2 was predicted under suitable conditions and for the one-dimensional case. Moreover, their results [6, 7] can be easy reproduced from our equation (6) when $\varepsilon_{\perp}$ is excluded from integration. Now the natural question arises: What is the reason for the discrepancy of our results with those of BB [4]? In our opinion such a discrepancy arises because of the infinite small resonance width approximation which was used in BB [4], when before their equation (28) they replaced at an intermediate stage of calculations the Breit-Wigner expression with a delta function. Moreover, when the Coulomb interaction is neglected, under this delta approximation the Fano factor found by BB [4] follows from equation $(8)$ of our work [1] $(\xi \ll-1)$. In other words, because of this delta approximation BB simply neglected the voltage interval where the Fano factor is below $1 / 2$.

Concerning the reasonability of the assumptions made, we confirm the use of energy independent tunnelling rates. We also confirm that current and noise are never exactly zero in the presence of an applied voltage. This is clear by carrying out a numerical calculation of the transparency for the structure drawn in the Comment. There is always nonzero transparency for electrons with energy above the band edge even when the resonant level is below it. Thus, the conclusion concerning the absence of the current and noise made by BB and used in their equation (14) [4] when the resonant level is below the band edge of the emitter is again the result of an approximation and does not correspond to a rigorous quantum mechanical description of tunnelling. Therefore, we disagree that by including an energy dependent tunnelling rate there are regions of applied voltages with exactly zero current and zero noise, unless one is forcing this result. We also disagree that finding a Fano factor below $1 / 2$ is strictly related to this approximation. As it is clear from figure 2 of our paper [1], there is wide region up to $\xi=-10$ for $f=\infty$ and $f=15$ where Fano factor is below $1 / 2$. In this region the resonant level is situated higher than the band edge of the emitter and the energy difference between them is greater than $\Gamma$, thus the influence of the edge on $\Gamma_{\mathrm{L}}$ is neglibible in calculating the transparency. It means that the Fano factor can drop below $1 / 2$ in the case of an energy dependent $\Gamma_{\mathrm{L}, \mathrm{R}}$ if in any point of the mentioned region of $\xi$ values $\Gamma_{L}=\Gamma_{R}$. As stated in the letter, we believe that in the coherent regime it is the presence of the (1-D) factor ( $D$ being the transparency of the device) which allows for a suppression below $1 / 2$, in analogy with the original findings of Lesovik [8] for the case of a point contact.

We agree that the simplicity of the model can be a source of discrepancy between experiments and theory, and that more investigations are needed to shed new light on the subject. We are presently engaged in this effort.

Concerning the inclusion of Coulomb effects, we presented as equation (6) in [1], we never found negative values of the current spectral density. On the other hand, we agree that the role of Coulomb interaction in general, and at zero temperature in particular, should be reconsidered more accurately than reported in equation (6). To this purpose we are working out a theoretical model following a full quantum mechanical approach [9]. In any case, at present we have not found results which contradict the essential findings reported in the letter.

\section{Acknowledgments}

Partial support from the Italian Ministry of Foreign Affairs (MAE) through the Volta Landau Center (the fellowship of VYaA) and from the cofin- 03 project 'Modelli e misure di rumore in nanostrutture' financed by the Italian Ministry for Education University and Research (MIUR) is gratefully acknowledged.

\section{References}

[1] Aleshkin V Ya et al 2003 Semicond. Sci Technol. 18 L35

[2] Iannaccone G, Macucci M and Pellegrini B 1997 Phys. Rev. B $\mathbf{5 5} 4539$

[3] Aleshkin V Ya and Reggiani L 2001 Phys. Rev. B 64245333

[4] Blanter Y M and Büttiker M 1999 Phys. Rev. B 5910217

[5] Blanter Y M and Büttiker M 2000 Phys. Rep. B 3361

[6] Averin D V 1993 J. Appl. Phys. 732593

[7] Wei Y, Wang B, Wang J and Guo H 1999 Phys. Rev. B 60 16900

[8] Lesovik G B 1989 JETP Lett. 49

[9] Aleshkin V Ya et al 2003 Preprint cond-mat/0304077 